\title{
Is routine pathological examination necessary in appendectomy for acute appendicitis?
}

\author{
J. Martellucci • A. Fontani • G. Tanzini
}

Accepted: 2 April 2009 /Published online: 25 April 2009

(C) Springer-Verlag 2009

Dear editor,

Appendectomy is still one of the most common emergency abdominal operations and is generally performed for a suspected diagnosis of acute appendicitis.

Although routine pathological analysis has shown a low incidence of detecting clinically significant unsuspected diseases, cancer of the appendix is an uncommon disease that is rarely suspected before surgery. Appendiceal neoplasm are reported in less than $2 \%$ of appendectomies and are typically associated to an acute appendicitis symptomatology and discovered only during operation or later during pathological examination. In fact, the majority of surgeons send all the surgical specimens for pathological examination.

It is also true that recent evidences suggest that many pathological analyses could be omitted for certain routine samples, and these kinds of examination are expensive and need time to be performed. Economic questioning of routine diagnostic procedures, especially pathological evaluation, is becoming increasing popular, aiming for a more responsible and evidence-based use of these procedures. A lower cost for a diagnostic test may be obtained by limiting its demand with a lower number of unnecessary specimens sent.

In 1996, the US College of American Pathologist Surgical Pathology Committee recommended the selection

J. Martellucci $(\bowtie) \cdot$ A. Fontani $\cdot$ G. Tanzini

General Surgery I, University of Siena,

Viale Bracci 1,

53100 Siena, Italy

e-mail: Jamjac64@hotmail.com of surgical resection specimens for examination, preferably with the creation of an institutional practice policy with two lists: one with specimens exempt from routine submission to the pathology and another with specimens for gross examination only.

On the other side, the common behavior responds to a social need, originated from the "defensive medicine" that surgeons have to consider in every performed action involving patients.

We retrospectively evaluated 1,219 consecutive patients with more than 18 years treated with appendectomy between 1986 and 2006. All the patients had a preoperative diagnosis of acute appendicitis. None of the patients had a preoperative diagnosis of appendiceal neoplasm. In all the patients, resected specimens were sent to pathologist for examination.

In 17 patients $(1.39 \%)$, appendix neoplasms were identified. Seven patients were found to have a mucocele or a cistoadenoma or the mixed form. In four patients, a mucinous adenocarcinoma was found with one case of pseudomyxoma peritonei. Other four patients were found to have carcinoid tumors and two patients had lymphoma. Crohn's disease was identified in four more patients. Carcinoids presented at an earlier mean age than the others. In all the patients, there was a suspicious macroscopic appearance.

Even if relevant histological findings after appendectomy are uncommon and the discovery of an unsuspected diagnosis in front of a macroscopically normal specimen is extremely rare, the histopathological analysis could not always be omitted, especially in those cases with a suspicious macroscopic appearance. 